\title{
SD-OCT in evaluation of microstructural changes in macula following blunt ocular trauma
}

\author{
Suryaprakash A.V ${ }^{1}$, Sunil Ganekal ${ }^{2}$, Vishal Korwar ${ }^{3, *}$, Shruti Choudhari ${ }^{4}$ \\ ${ }^{1}$ Professor \& Unit Chief, ${ }^{2}$ Associate Professor, ${ }^{3,4}$ Post Graduate Student, Dept. of Ophthalmology, J.J.M. Medical College, \\ Davangere, Karnataka, India
}

*Corresponding Author

Email: vishalkorwar23@gmail.com

\begin{abstract}
Introduction: Visual morbidity due to blunt ocular trauma (BOT) is common but there is paucity of reports on posterior segment manifestations following BOT using Spectral domain optical coherence tomography (SD-OCT). In this study, an attempt is made to assess the microstructural changes in macula following BOT using SD-OCT.

Objectives: To evaluate microstructural changes in macula following blunt ocular trauma.

Materials and Methods: A prospective study conducted on 30 patients with BOT of any age and gender attending Ophthalmology outpatient department and casualty at Hospitals attached to JJM Medical College, Davangere. Detailed history of trauma and thorough examination was done. Patients were evaluated for macular changes using SD-OCT at presentation and follow up at 1 and 6 months from trauma.

Results: In this study, $19(63.3 \%)$ patients had changes involving the fovea and 11(36.6\%) had foveal sparing changes. 14(46.6\%) eyes had commotion like changes, 4 (13.3\%) eyes developed macular hole, 2(6.6\%) eye had choroidal rupture passing through the fovea, $1(3.3 \%)$ eye had retinal detachment. 11(36.6\%) patients had extrafoveal involvement in the form of choroidal ruptures in 5(16.6\%) eyes and significant RPE alterations sparing fovea in 6(20\%) eyes.

Conclusion: OCT plays a vital role in detecting macular microstructural changes. Thus OCT can be useful in evaluation and management of BOT.
\end{abstract}

Keywords: Blunt ocular trauma, Macular changes, SD-OCT.

\section{Introduction}

Blunt ocular trauma may cause a variety of posterior segment abnormalities causing visual morbidity due to microstructural changes in the retinal architecture. BOT may cause commotio retinae, retinal pigment epithelial edema, choroidal rupture and optic nerve evulsion alone or in combination. Traumatic macular holes and retinal detachment or dialysis may also occur after BOT. ${ }^{1}$

Optical coherence tomography (OCT) is a noninvasive optical imaging technique that provides high-resolution, cross-sectional, in-vivo imaging of the human retina from which estimates of retinal layers can be made. ${ }^{2}$

Optical coherence tomography is of great value in the management of patients with posterior segment trauma. It defines anatomic details to confirm the diagnosis and may allow for a better understanding of the pathogenesis, especially in acute traumatic maculopathy. ${ }^{3}$

Though visual morbidity due to blunt ocular trauma is quite common, there is paucity in reports conducted using SD-OCT to study the microstructural changes in macula.

Spectral-domain OCT was used in our study to describe the morphologic characteristics of post traumatic macular changes and to evaluate its utility in prognosis and follow-up.

\section{Materials and Methods}

1. This prospective protocol adhered to the tenets of the Declaration of Helsinki.

2. A written informed consent was obtained from all subjects.

Inclusion Criteria: 30 patients with blunt ocular trauma of any age and gender attending Ophthalmology Outpatient Department and Casualty at Bapuji Hospital and Chigateri General Hospital attached to JJM Medical College, Davangere.

\section{Exclusion Criteria:}

1. Patients with penetrating injury to eye.

2. Patients with lens and media opacities.

3. Patients with traumatic glaucoma.

Procedure: The data for this study was collected from the subjects who fulfilled the inclusion /exclusion criteria.

A detailed history of trauma was recorded. All the patients were evaluated at presentation (within a week) following blunt ocular trauma with follow up at 1 and 6 month from the day of trauma. At each visit patients were evaluated as follows:

1. Best corrected visual acuity: using Snellen's chart and converted to $\log$ MAR units.

2. Slit lamp examination, intraocular pressure with Goldmann applanation tonometry.

3. Fundus evaluation using direct ophthalmoscope and indirect ophthalmoscope.

4. Macular cube analysis was done by using Zeiss CIRRUS ${ }^{\mathrm{TM}}$ HD OCT (500) through a dilated pupil. 
Three of the best obtained scans were selected with quality of scans set at signal strength more than 7 .

\section{Statistical Analysis}

1. Qualitative data was represented in the form of frequency and percentage.

2. Quantitative data was represented using mean \& Sd. Analysis of quantitative data between two groups was done using unpaired t test if data passes 'Normality test'.

3. Analysis was done with IBM SPSS version 22 for Windows.

\section{Results and Discussion}

Patient's age ranged from 18 to 38 years with mean age of 25.93 years.

Majority of the patients $18(60 \%)$ were aged between 20 to 29 years. Among 30 patients $28(93.3 \%)$ were males and $2(6.7 \%)$ were females.

Most of the patients $18(60 \%)$ sustained trauma to right eye and the remaining patients 12 (40\%) had left eye involvement.
Out of 30 patients $15(50 \%)$ patients had trauma with the objects larger than the orbit, $9(30 \%)$ patients had trauma with objects smaller than orbit, whereas $6(20 \%)$ sustained trauma following road traffic accident.

Average BCVA at presentation, 1 month and 6 month was $0.51,0.38$ and $0.21 \log$ MAR units respectively. (Table 1)

Table 1: Average BCVA

\begin{tabular}{|l|c|c|}
\hline BCVA & $\begin{array}{c}\text { Mean (log MAR } \\
\text { units) }\end{array}$ & Std. Deviation \\
\hline Basal & 0.51 & 0.64 \\
\hline 1 Month & 0.38 & 0.64 \\
\hline 6 Month & 0.21 & 0.35 \\
\hline
\end{tabular}

Macular involvement was either foveal or extrafoveal. $19(63.3 \%)$ patients had changes involving the fovea and $11(36.6 \%)$ had foveal sparing changes. 2 patients with commotio retinae also presented with coexisting choroidal rupture and macular hole each. (Table 2)

Table 2: Frequency of macular changes

\begin{tabular}{|l|c|c|c|c|c|}
\hline \multirow{2}{*}{ Macular changes } & \multicolumn{2}{|c|}{ Foveal } & \multicolumn{2}{c|}{ Extra foveal } & \\
\cline { 2 - 6 } & Frequency & Percentage & Frequency & Percentage & Total \\
\hline Commotio retinae & 14 & 46.6 & 0 & 0 & 15 \\
\hline Choroidal rupture & 2 & 6.6 & 5 & 16.6 & 7 \\
\hline Macular hole & 4 & 13.3 & 0 & 0 & 4 \\
\hline RPE changes & 0 & 0 & 6 & 20 & 6 \\
\hline Retinal detachment & 1 & 3.3 & 0 & 0 & 1 \\
\hline
\end{tabular}

Commotio Retinae: $14(46.6 \%)$ patients had commotio like changes involving fovea with altered foveal reflex noted in 5 eyes. Along with foveal involvement mid peripheral commotio was seen in 7 eyes.

On OCT, prominent features in eyes with commotio were change in reflectivity of the retinal layers with variable increased reflectivity from the inner retinal layers, increased reflectivity of the ISOS junction. At a later stage these reflectivity changes resolved and became imperceptible.

Choroidal Rupture: In this study 7 (23.33\%) eyes had choroidal ruptures of which $2(6.6 \%)$ were involving fovea and $5(16.6 \%)$ were extrafoveal. 2 eyes had peripheral choroidal ruptures and 2 eyes had peripapillary choroidal rupture.

On OCT, there were two patterns identifiable in choroidal ruptures, type 1 pattern was seen in 4 of the chororidal ruptures, type 2 was seen in 3 eyes. (Table 3 )

Table 3: Choroidal rupture

\begin{tabular}{|l|c|c|}
\hline \multicolumn{3}{|c|}{ Macular changes } \\
\hline Choroidal rupture & Foveal & Extra foveal \\
\hline Type 1 & 0 & 4 \\
\hline Type 2 & 2 & 1 \\
\hline
\end{tabular}

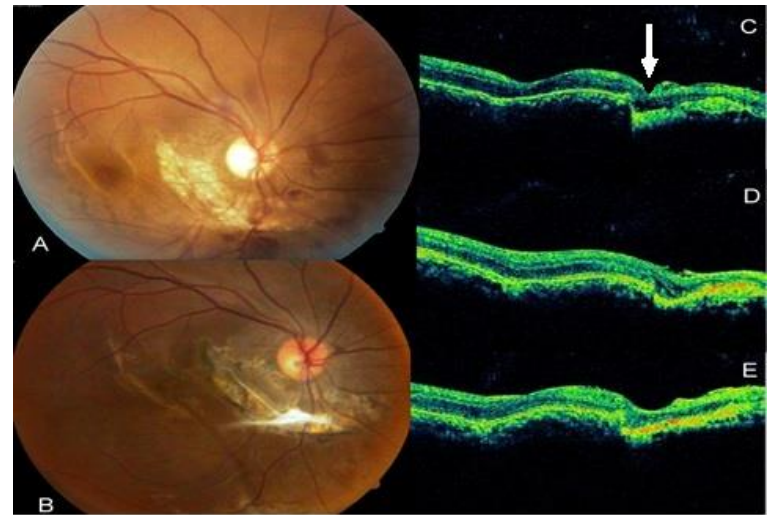

Fig. 1: A. Perifoveal and peripapillary choroidal rupture with preretinal hemorrhage at presentation; B. Scaring with fibrosis at 6 month; C. Perifoveal choroidal rupture type-2 at presentation with ISOS disruption; D. \&E. Increased reflectivity owing to fibrosis and scaring at $1 \& 6$ month respectively)

Macular Hole: In this study 4 eyes had macular hole of which 3 were lamellar holes and 1 was full thickness. 1 was associated with peripapillary hemorrhage and 1 with pigment epithelial detachment and commotio retinae. 
On OCT, partial thickness defect of outer retina at fovea with intact inner layers was seen in 3 eyes with lamellar hole and full thickness defect measuring $110 \mu$ was seen in 1 eye. 2 eyes with lamellar macular hole showed resolution with spontaneous closure. 1 eye had persistent hole on follow up. 1 eye with full thickness macular hole underwent vitrectomy with ILM peeling and perfluoropropane infusion and showed good visual recovery.

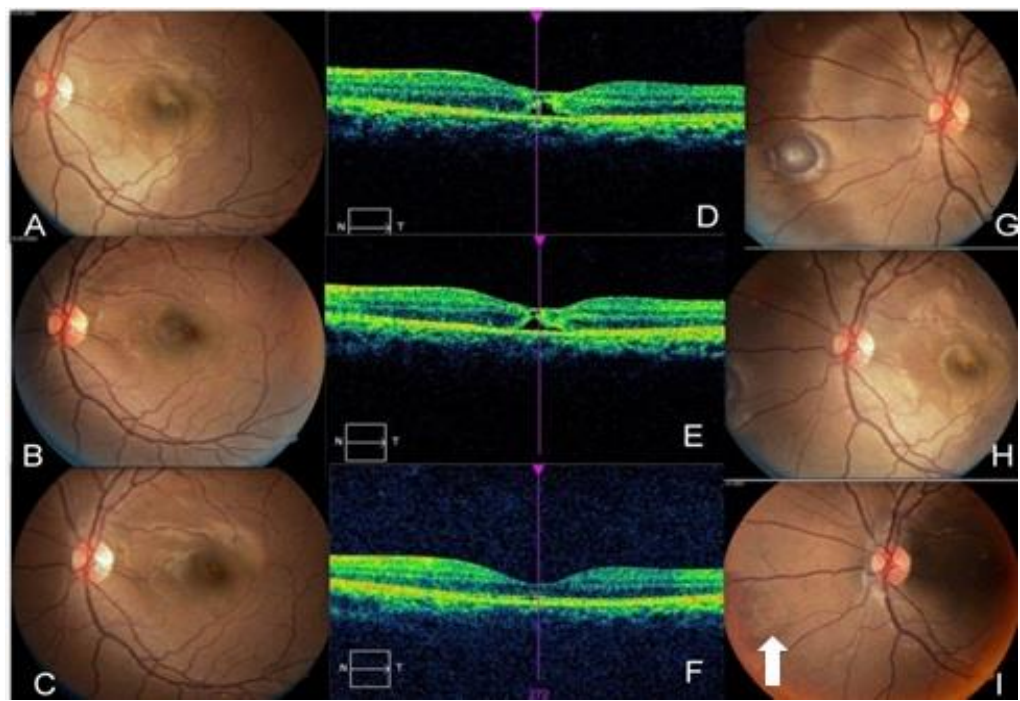

Fig. 2: A. Well demarcated \& hyperpigmented lession at macula with commotio at presentation; B. \& C. resolving hyperpigmentation \& commotio over 6 months; D. to F. spontaneous resolution and closure of outer lamellar hole over 6 months. G) to I) associated PED with resolution over 6 months)

RPE Alterations: 6(20\%) patients had RPE changes presented with yellowish hypopigmentation in macula sparing the fovea.

On OCT, they revealed area suggestive of outer lamellar translucency which was likely to correspond to an area of photoreceptor disruption. Over the time the area of outer retinal lucency subsided resulting in an area of loss of ISOS junction.

Retinal Detachment: In this study, 1 patient developed subfoveal retinal detachment with peripheral retinal break which on OCT revealed separation of neurosensory retina from the RPE. Patient was treated with scleral buckle and showed moderate visual recovery.

Associated Features: (Table 4) Among 19 patients with foveal involvement, $14(46.6 \%)$ eyes had commotio like changes which is consistent with the study of $\mathrm{Oh} \mathrm{J}$ et $\mathrm{al}^{4}$ which reported $50 \%$ eyes had developed commotio retinae following blunt ocular trauma. Unnikrishnan et $\mathrm{al}^{5}$ reported commotio involving fovea in $27.77 \%$ eyes.

Table 4: Associated features

\begin{tabular}{|l|c|c|}
\hline \multicolumn{1}{|c|}{ Associated findings } & Frequency & Percentage \\
\hline Pre-retinal hemorrhage (PRH) & 3 & 13.3 \\
\hline Subretinal hemorrhage (SRH) & 3 & 13.3 \\
\hline Peripapillary hemorrhage (PPH) & 2 & 6.7 \\
\hline Peripapillary choroidal rupture (PPCR) & 2 & 6.7 \\
\hline Peripheral choroidal rupture (PCR) & 2 & 6.7 \\
\hline Pigment epithelial detachment (PED) & 1 & 3.3 \\
\hline Peripheral retinal break(PRB) & 1 & 3.3 \\
\hline
\end{tabular}

Studies of OCT on commotio retinae by Joo Youn Park et al, ${ }^{6}$ Meyer $\mathrm{CH}$ et $\mathrm{al}^{7}$ and Sony $\mathrm{P}$ et $\mathrm{al}^{8}$ revealed transient abnormalities at the level of photoreceptor segments in form of disruption and fragmentation which was similarly noted in this study. Leila El Matri et $\mathrm{al}^{3}$ observed that increased reflectivity on OCT in berlin's edema represents photoreceptor outer segment disruption which is consistent with our study. In this study all the eyes with commotio had good visual recovery which was similarly noted by Leila El Matri et al. ${ }^{3}$

In this study $4(13.3 \%)$ eyes had macular hole of which 3 were lamellar holes and 1 was full thickness. The study by Querques $\mathrm{G}$ et $\mathrm{al}^{9}$ reported that frequency of traumatic macular holes (TMH) is between $1 \%$ and $9 \%$ which is similar with our findings. 
Among 3 eyes with lamellar hole, 2 showed resolution, 1 eye had persistence of hole and 1 FTMH was treated surgically. Previous studies have utilized OCT imaging in documenting the natural progression and spontaneous closure of traumatic macular holes anatomically. ${ }^{5}$

In this study $7(23.33 \%)$ eyes had choroidal ruptures of which $2(28.5 \%)$ were involving fovea and 5 were extrafoveal, 2 eyes had peripapillary choroidal rupture and 2 eyes had peripheral choroidal ruptures. In a study by Unnikrishnan et $\mathrm{al}^{5} 8(44.4 \%)$ had choroidal rupture out of which $2(11.11 \%)$ passed through the fovea which was not similar to our findings. In a study conducted by Wood et $\mathrm{al}^{10} 5$ of $30(17 \%)$ eyes had choroidal ruptures through the fovea.

On OCT, There were two patterns identifiable in choroidal ruptures, the first being a forward protrusion of the RPE-CC layer (Retinal pigment epitheliumchoriocapillaries) with an acute pyramidal or dome shape. This pattern was seen in five of the choroidal ruptures. The second pattern seen was a large disruption of the RPE -CC, ISOS and ELM layer (External limiting membrane) with a depression at this area and downward sliding of tissues into the defect. This pattern was seen in two eyes. These findings were consistent with the reports of Unnikrishnan et al. ${ }^{5}$

In this study $7(23.33 \%)$ eyes with choroidal rupture involving macula showed poor visual recovery which was similar with the findings of Wood et $\mathrm{al}^{10}$ who noted that $10(33.33 \%)$ eyes had impaired visual acuity owing to pigmentary changes involving fovea or photoreceptor loss.

In this present study $6(20 \%)$ patients had RPE changes and presented with yellowish hypopigmentation in macula sparing the fovea. Which is consistent with the results of Unnikrishnan et $\mathrm{al}^{5}$ who reported that 3(16.66\%) eyes had RPE alterations. They also reported that severe retinal damage, which led to profound and long-lasting visual impairment was attributed to acute loss or attenuation in the ISOS junction as appeared on SD-OCT. But in this study patients had good visual recovery owing to mild retinal damage and foveal sparing RPE alterations.

In this study, 1 (3.3\%) patient developed subfoveal retinal detachment with peripheral retinal break which on OCT revealed separation of neurosensory retina from the RPE. Similarly Sujatha M et al reported that retinal detachment following blunt ocular trauma was seen in $2 \%$ of eyes. In this study Patient was treated surgically with scleral buckle and showed moderate visual recovery over the time.

All patients were treated according to standard medical protocol. Most of them responded well to oral steroids and showed good visual recovery.

\section{Conclusion}

Although the small sample of patients studied and variable follow-up period of this study, SD-OCT proved to be a useful tool in the evaluation of patients with BOT. Study findings represent a spectrum of lesions, depending on the intensity of the trauma. Most of the foveal involvement was in the form of commotio retinae whereas other findings included maular holes, choroidal ruptures and retinal detachment. Extrafoveal involvement was in form of choroidal ruptures and RPE alterations. Majority of patients had good visual recovery except those with severe foveal damage with poor prognosis which was accounted by using SDOCT.

Thus, OCT is vital tool in detecting subtle microstructural changes and therefore helps in evaluvation and management of posterior segment manifestations following blunt ocular trauma.

\section{References}

1. Williams DF, Mieler WF, Williams GA. Posterior segment manifestations of ocular trauma. Retina. 1990; 10 Suppl 1:S35-44.

2. R M Vessani, L P Cunha and M L R Monteiro. Progressive macular thinning after indirect traumatic optic neuropathy documented by optical coherence tomography. Br J Ophthalmol. 2007 May; 91(5):697-698.

3. Leila El Matri, Ahmed Chebil, Fedra Kort, Rym Bouraoui, Leila Largueche, Fatma Mghaieth . Optical Coherence Tomographic Findings in Berlin`s Edema. $J$ Ophthalmic vis Res. 2010 April;5(2):127-129.

4. Oh J, Jung JH, Moon SW, Song SJ, Yu HG, Cho HY. Commotio retinae with spectral-domain optical coherence tomography. Retina. 2011 Nov;31(10): 2044-9.

5. Dr. Unnikrishnan Nair R. Assessment of macular involvement in blunt trauma using SD OCT and multifocal ERG. 70 ${ }^{\text {th }}$ AIOC Proceedings, Cochin 2012. Available from URL http://www.aiosedu.org/uploads/BOB17.pdf

6. Park JY, Nam WH, Kim SH, Jang SY, Ohn YH, Park TK. Evaluation of the central macula in commotio retinae not associated with other types of traumatic retinopathy. Korean J Ophthalmol. 2011 Aug;25(4):262-7.

7. Meyer CH, Rodrigues EB, Mennel S. Acute commotio retinae determined by cross-sectional optical coherence tomography. Eur J Ophthalmol. 2003 Nov-Dec;13(910):816-8.

8. Sony P, Venkatesh P, Gadaginamath S, Garg SP. Optical coherence tomography findings in commotio retina. Clin Exp Ophthalmol. 2006 Aug;34(6):621-3.

9. Querques G, Barone A, Forte R, Prascina F, Iaculli C, Delle Noci N: Optical coherence tomography and fundusrelated perimetry in spontaneous closure of a traumatic macular hole. J Fr Ophtalmol. 2008, 31: 710-713.

10. CM Wood and J Richardson Indirect choroidal ruptures: aetiological factors, patterns of ocular damage, and final visual outcome. Br J Ophthalmol. 1990;74:208-211.

How to cite this article: Suryaprakash AV, Ganekal S, Korwar V, Choudhari S. SD-OCT in evaluation of microstructural changes in macula following blunt ocular trauma. Ind J Clin Exp Ophthalmol. 2018;4(3):413-416. 